Revue d'histoire de l'Amérique française

ZWB REVUE D.HISTOIRE DE L'AMÉRIQUE FRANÇAISE

\title{
Le Syndic de la colonie acadienne en Poitou (suite)
}

\section{Pierre Massé}

Volume 5, numéro 2, septembre 1951

URI : https://id.erudit.org/iderudit/801700ar

DOI : https://doi.org/10.7202/801700ar

Aller au sommaire du numéro

Éditeur(s)

Institut d'histoire de l'Amérique française

ISSN

0035-2357 (imprimé)

1492-1383 (numérique)

Découvrir la revue

Citer cet article

Massé, P. (1951). Le Syndic de la colonie acadienne en Poitou (suite). Revue

d'histoire de l'Amérique française, 5(2), 252-264.

https://doi.org/10.7202/801700ar d'utilisation que vous pouvez consulter en ligne.

https://apropos.erudit.org/fr/usagers/politique-dutilisation/ 


\title{
LE SYNDIC DE LA COLONIE ACADIENNE EN POITOU* (suite)
}

\author{
III
APPARITION D'UN NOUVEAU PAYSAGE RURAL: ACTION DU SYNDIC

Que l'on ait pu introduire la complexité dans la mosaïque simpliste du géomètre, enchevêtrer cet écheveau, effacer quelquesunes des lignes si bien tirées au cordeau: un tel fait a droit de surprendre. C'est oublier que le laboureur est, au premier chef, un créateur de terroir. "Dès que l'homme travaille, dès qu'il pense, le paysage porte sa marque, et parfois une marque compliquée"68. Le plateau de Monthoiron, socle de la colonie, n'échappera pas à cette formule.

Vincent Amirault, toutes les fois que la chose était possible, avait tracé des métairies d'un seul tenant. Vieille loi agraire. Les labours, prés et bois unifiés en un bloc solide autour des bâtiments d'habitation, se constatent chaque fois que le fondateur du domaine a devant lui, par conquête, achat ou défrichement, un espace où il peut tailler à sa guise. Telle est la villa du Haut Moyen Age, succédant parfois à la villa gallo-romaine, et dont la survivance se transmet jusqu'au XVIIIe siècle ${ }^{69}$. La seigneurie de Varennes, à Bonneuil-Matours se présente ainsi en l'an II. "Tout cet ensemble forme un seul mas"70. Telles sont, dans la même commune, les métairies de la Jarlandière, des grandes et petites Couinières, cisaillées à l'emporte-pièce à travers les étendues de brandes ${ }^{71}$. Telles furent

* Voir, Revue d'Histoire de l'A mérique française, vol. V, no 1: 45-68.

68. André Allix, L'homme dans la géographie humaine, et le problème du libre aroitre (L'Education Nationale, Paris, 1950), no 9, 3 a 5).

69. Charles-Edmond Perrin, Recherches sur la seigneurie rurale en Lorraine a'après les plus anciens censiers (IXe-XIIe siècles) (Strasbourg, 1935). - Philippe Dollinger, L'évolution des classes rurales en Bavière depuis la fin de l'époque carolingienne jusqu'au milieu du XIIIe siècle (Paris, 1950).

70. Archives de la Vienne. Q2, 43.

71. Archives de la Vienne. H 41. 
15 des métairies acadiennes sur les 57 que le géomètre eut à délimiter. Pour les autres, dont le morcellement se limitait à 2 ou 3 tronçons, et n'atteignait qu'une seule fois 4 , il éprouvait le besoin d'expliquer et presque d'excuser cette disposition:

"L'irrégularité de quelques-unes des pièces de terre comprises au présent plan résulte de ce que la position de plusieurs maisons de la dite colonie ne s'est pas trouvée en rapport avec le terrain qui est le plus proche des dites maisons, et de ce que les personnes de la dite colonie, n'ayant pas eu de limites respectives depuis la fixation, ont défriché, labouré et amélioré du terrain qui pouvait convenir aux uns et non aux autres, mais qui leur est demeuré, par les susdites opérations, comme l'ayant exploité"72.

Amirault n'écrit pas comme Stendhal, mais on n'en comprend pas moins sa déclaration. Elle laisse supposer que l'arpentage fut accompagné de négociations qui expliquent en partie, avec les incidents que nous avons vus, la longue durée de son cours. On ne saurait garder trop de gratitude à Papuchon, dont le plan illustre, de façon frappante, la pensée directrice de l'entreprise. Eût-il borné son ouvrage à la publication de cette pièce, découverte, dit-il, au presbytère de La Puye en 1902, que le général eût apporté, à l'histoire acadienne, une contribution de première grandeur. Le domaine de Texier-Latouche, afférent aux No 17 et 18, y apparaît, réparti en trois pièces dont deux se touchent, la troisième enclavée entre des riverains.

Il sera d'un haut intérêt de suivre les modifications de ce plan, auquel les paysans auront tôt fait de s'attaquer. Ils vont le reprendre, le remanier, le pétrir, jusqu'à rendre parfois méconnaissable le dessin initial, supplanté par d'autres dessins inspirés des coutumes millénaires. Nous sommes en pays d'openfield, soumis à l'assolement triennal. Vont apparaître bien vite, par-dessus la belle ordonnance d'Amirault, les champs ouverts et irréguliers caractéristiques de la région. Si la colonie s'était installée dans une lande du Bocage, nous la verrions bientôt découpée en champs carrés, bordés de haies et de chemins creux $^{73}$. Expérience unique en son genre,

72. Papuchon, La colonie acadienne du Poitou (Poitiers, 1908).

73. Voir, à ce sujet, l'exemple très caractéristique cité par Dion: Essai sur la formation du paysage rural français (Tours, 1934), 65 à 69. 
avons-nous dit en commençant. Les familles acadiennes vont transformer le paysage rural en refaisant, presque sous nos yeux, ce que leurs voisins d'Archigny, Monthoiron, La Puye, Bonneuil-Matours, ont mis des siècles à accomplir. Brulant les étapes que la Révolution agricole du XIXe siècle leur permet d'abréger encore, ils fabriqueront de toutes pièces un pays.

Et leur syndic, quelque fidélité qu'il pât garder à une cause devenue la sienne, en vint à saper lui-même son œuvre propre. Il fut le premier à dénaturer les carrelages qu'il avait eu tant de mal à faire réaliser. Il marche en tête de l'équipe où l'on reconnaît, au coude à coude, Acadiens et gens du pays, qui vont démolir l'édifice si laborieusement échafaudé, pour le reconstruire ensuite à leur idée.

Un nom très répandu parmi les Acadiens est celui d'Hébert, vieille famille souche où il est difficile de préciser les parentés ${ }^{74}$. Deux colons, Etienne Hébert et Marie Lavergne, baptisent un fils, Louis Gabriel, le 20 juin 1775, à Cenant ${ }^{75}$. On retrouve d'autres Hébert dans le convoi parti de Châtellerault pour Nantes, le 15 novembre suivant ${ }^{76}$. Or, deux filles d'un Jean-Baptiste Hébert, décédé, demeurèrent en Poitou. L'une, Anne, épousa François Martin, laboureur à Cenant, le 27 aout $1776^{77}$. La plus jeune, Marie, étant mineure, passa sous la tutelle de son beau-frère. Pérusse d'Escars projetait de lui accorder le No 19, en 1784, tandis que François Martin et Anne Hébert obtenaient le No 24.78.

Le syndic ne pouvait manquer d'être intéressé par ce No 19, qui avoisinait directement ses propriétés et celles du sieur Berbudeau. Très tôt, il cherchait à le louer. François Martin, tuteur de Marie Hébert, devait être suffisamment occupé par la mise en valeur de son No 24, à Cenant, pour mener de front les deux exploitations.

74. Edmond Richard, Acadie (3 vols., Québec, 1916), I: 49.

75. Regentes paroissiaux de Cenant.

76. Archives de la Vienne, dépôt 22, liasse 124.

77. Registres paroissiaux de Cenant.

78. Archives de la Vienne, dépôt 22, liasse 98. 
Le 2 décembre 1785, Pierre Alexis Texier de la Touche, bourgeois, obtenait un bail de la maison et des terres du No 19, consenti par Martin au nom de sa pupille. Bail de 9 ans, commençant à la Saint-Martin précédente et fait "à titre de ferme à prix d'argent et non autrement". Entrons un peu dans les détails, où nous allons voir apparaître des différences notables avec les autres baux de la région.

Un peu partout, en effet, dans le pays environnant, les actes notariés comprennent, aux XVIIe et XVIIIe siècles, des baux à colonnage, c'est-à-dire avec partage des récoltes à moitié entre le propriétaire et le métayer. Mais ici, entre en scène un facteur nouveau: le défrichement. Le No 19 ne semble guère comporter qu'un jardin - dont Martín se réserve une boisselée, avec une chambre à feu dans la mạison, - et sans doute une certaine surface essartée, qui n'est malheureusement pas connue. Texier s'engage à défricher par an une bơisselée et demie de brandes qu'il marnera aussitôt. Un simple calcul nous indique qu'il aura défoncé à la fin de son bail 13 boisselées 1/2. Autrement dit l'étendue de ce que l'on appelle une borderie, ou métairie en réduction.

Le loyer expirant au bout de 9 ans, soit en 1794, la borderie sera créée. Alors, le système du colonnage pourra fonctionner. Texier ensemencera "au mois d'octobre de.la dernière année du bail, en 1794, le tiers des terres labourables, et les fruits de la récolte 1795 seront partagés par moitié entre le bailleur et le preneur". Le No 19 prendra place parmi les autres machines à blé du pays: un tiers en blé d'hiver, un tiers en blé de printemps, un tiers en jachère, les brandes non défrichées servant de pacage.

Quant au prix de ferme, en attendant que le colonnage puisse être appliqué, il sera progressif, s'élevant à mesure que les défrichements s'étendent et donnent au locataire des revenus accrus. Il sera de 18 livres pour chacune des 3 premières années, 24 livres pour chacune des 6 dernières ${ }^{79}$. François Martin avait loué des terres incultes; il retrouvera une borderie d'environ 13 boisselées et susceptible d'agrandíssement. L'affaire, au départ, s'annonce bien pour le bailleur. Mais que vaudront les 24 livres perçues en 1793 et 1794 ? Le laboureur de Cenant ne pouvait pas prévoir l'ef-

79. Minutes Amirault. 
fondrement des assignats. Sa tutelle, par ailleurs, va bientôt prendre fin. La jeune Marie Hébert a trouvé un mari en la personne du maréchal François Charaudeau, veuf de Marie Robin, de Leigné-lesBois, qui l'épouse le 9 février $1790^{80}$.

Jusqu'ici, les faits se laissent suivre assez facilement. Cependant, il est une notion qu'il faut avoir toujours présente à l'esprit toutes les fois que l'on s'occupe des Acadiens avant le bornoiement de 1793: celle de l'imprécision des titres de propriété, si titre il y a. En ce qui concerne le No 39, nous apprenons, avec un étonnement que l'habitude commence à émousser, que le domaine n'appartenait pas à Marie Hébert quand son tuteur le donna en location au syndic, mais à la demoiselle Pérusse d'Escars. Il figure, en effet, parmi les métairies attribuées à la fille du marquis par le Conseil d'État, le 26 juillet 1785 . Nous avons vu que le No 40 avait deux propriétaires: Guillard de la Vacherie; La Bussière et Champagne. Le No 19 était dans la même situation paradoxale. Comment avait-on pu en arriver là?

Les notes laissées par Pérusse d'Escars éclairent un peu la question. Elles révelent que les projets d'attribution des habitations, mis noir sur blanc par le marquis, ont subi des remaniements successifs, après les départs des mécontents pour Nantes, et les décès survenus chez les colons fidèles. Il y eut des échanges et des reprises, comme nous l'avons constaté pour René Baudeau. "J'ai donné à Marie Hébert le No 19 qui était vacant", écrit Pérusse à deux reprises sur des notes non datées ${ }^{81}$. Possession vaut titre, mais la description du No 19, lorsque Martin le loue à Texier, nous montre que la possession, dans les mains du tuteur, n'avait pas été poussée bien loin.

La sœur de Marie Hébert, qui a épousé François Charaudeau, n'est pas mieux partagée. L'ancêtre acadien, Jean-Baptiste Hébert, s'était vu attribuer le No 23, à Cenant. Il y avait travaillé ferme et défriché 130 boisselées, dont 36 étaient marnées quand il mourut. Il pouvait s'y croire chez lui, et son gendre, le maréchal de Leignéles-Bois, ne pensait probablement pas qu'il en serait un jour évincé. Ce fut pourtant chose faite, le jour où le Conseil d'Etat donna le

80. Registres paroissiaux d'Archigny.

81. Archives de la Vienne. dépôt 22, liasse 94. 
No 23 à la jeune châtelaine. Si bien que les habitations affectées au deux sœurs acadiennes leur étaient enlevées. Ce que le père avait donné, la fille le reprenait.

Une fois encore, le notaire intervint, et aussi Pérusse d'Escars, bien qu'il dât avoir, à cette date, des préoccupations plus vives. L'acte rédigé au château de Monthoiron, le 1er mars 1791, témoigne du souci de réparer des préjudices où le marquis avait quelques responsabilités. La demoiselle de Pérusse effectue un achat qui apparait comme le versement d'une compensation aux sœurs acadiennes, par l'intermédiaire de leurs époux. Ceux-ci lui vendent "les droits et prétentions, soit de propriété, de possession, soit d'indemnité pour défrichements, amélioration ou jouissance, qu'ils possèdent sur le No 23, qui avait été accordé à l'Acadien JeanBaptiste Hébert". Reconnaissance explicite des droits du premier occupant. Aveu, également, que les décisions du Conseil d'État ont été prises sans s'entourer des précautions indispensables. Par son achat, la demoiselle "remplace les héritiers Hébert dans tous leurs droits et privilèges", et, pour ce, leur verse 1200 livres à se partager par moitié.

Avant que l'on se sépare, François Martin et sa femme présentent une demande au marquis. Ils se trouvent extrêmement gênés pour la sortie de leurs bestiaux en l'habitation où ils demeurent, et qui est le No 24. Afin d'avoir un accès plus facile à leur exploitation, ils désirent acheter 30 boisselées de brandes dans son voisinage immédiat. Pérusse accèdera volontiers à cette demande, mais plus tard. Il faut d'abord que les limites des propriétés soient précisées. "Ayant consenti à l'établissement qu'il a plu au Roy de faire sur une partie de son terrain, il veut fournir, autant qu'il sera possible, nécessaire et convenable, les terres suffisantes à chaque habitation". Quand cette affaire sera réglée, il donnera volontiers satisfaction aux demandeurs ${ }^{82}$. Les circonstances ne lui permirent point de tenir ses engagements; il fallut que d'autres s'en chargent pour lui.

Ayant indemnisé François Martin et sa jeune femme, et promis de les avantager encore dans l'avenir, Pérusse d'Escars manifesta, envers l'autre ménage, moins de scrupules. Il leur abandonna le

82. Minutes Amirault. 
No 19, mais contre un versement de 400 livres $^{83}$. Disons, pour tenter d'expliquer cette différence de traitement, que le No 23 était alors exploité par un métayer du pays, Sylvain Martial, installé là par le marquis, et qui avait continué les défrichements de Jean-Baptiste Hébert. Ajoutons que l'exploitation était garnie de bestiaux, outils et instruments aratoires appartenant à Pérusse. Le No 19, par contre, était affermé par Charaudeau à Texier-Latouche, et rapportait déjà un certain revenu à son pseudo-propriétaire. Lorsque les questions paysannes se doublent de questions acadiennes, on arrive à des complications jamais atteintes dans l'histoire agraire de la région.

On pouvait croire ainsi réglées les questions jumelles des No 19 et 23. La Révolution, avec l'émigration du marquis, vint les faire rebondir.

Parti de France à la fin de juillet 1791, Pérusse d'Escars donna de ses nouvelles, au mois d'aout 1792, d'une manière qui fit sensation, en envoyant le manifeste de Brunswick, écrit de sa propre main, à la municipalité de Châtellerault ${ }^{84}$. Le maire de la ville apporta plus de retentissement encore au factum, qu'il recopia tout au long sur le régistre des délibérations ${ }^{85}$, maladresse qui le fit destituer avec fracas et faillit lui couter $\operatorname{cher}^{86}$. La vindicte nationale s'abattit sur les biens du marquis. Le château de Monthoiron et les terres dépendantes, mises sous séquestre, ne furent plus qu'une belle proie, promise aux prochains acheteurs.

La demoiselle Pérusse d'Escars, restée au pays, put alors se féliciter que le Conseil d'État de 1785 l'ait nommément désignée comme propriétaire de plusieurs habitations auxquelles personne ne pouvait prétendre. Personne? Au plus fort de la Terreur, le 24 floréal an II, elle dut faire face à une tentative d'intimidation qui ressemble étrangement a celle dont fut l'objet Texier-Latouche. Fille d'émigré, il n'est pas certain que les tribunaux l'eussent soutenue. Elle céda donc, et s'en tira encore à bon marché.

83. Ibid.

84. Archives Nationales F 1b, II, Vienne 1.

85. Archives municipales de Chatellerault. Registre de délibération.

86. Archives de la Vienne, L 272, reg. 6. 
Les deux héritières de Jean-Baptiste Hébert et leurs époux n'avaient sans doute pas accepté de gaieté de cœur la transaction du 1er mars 1791, leur enlevant les No 19 et 23 dont ils avaient de bonnes raisons pour se croire possesseurs légitimes. Le seigneur de Monthoiron disparu, et les aristocrates devenus suspects, François Martin et Anne Hébert estimèrent qu'ils avaient été lésés dans l'achat du No 23 par la demoiselle Pérusse d'Escars, celle-ci n'ayant pas payé la métairie le prix qu'elle valait réellement. Et de réclamer un dédommagement substantiel, pour la perte qu'ils avaient subie au temps où la noblesse pouvait encore parler le verbe haut.

Pour l'instant, cette classe sociale, dans la région, était bien touchée. Deux des plus représentatifs de ses membres attendaient d'être traduits devant le Tribunal révolutionnaire et de monter sur l'échafaud, en compagnie d'Emery Gauvain, le notaire de Vouneuil qui avait dressé en 1781, le contrat de mariage de Texier-Latouche et Marie Reine Berbudeau ${ }^{87}$. Ces arrestations avaient fait grand bruit. Appelvoisin de la Roche du Maine, seigneur du Fou, était un voisin et ami de Pérusse d'Escars. Aussi le marquis de Chasteigner, seigneur de Touffou, paroisse de Bonnes. Quant au notaire Gauvain, il était bien connu à Monthoiron, où il avait présidé à de nombreuses transactions. Le moment était mal venu pour demander à un magistrat d'examiner impartialement les intérêts de la fille d'Escars. Celle-ci, qui avait pour fondé de pouvoirs, depuis le 23 novembre 1791, c'est-à-dire depuis l'émigration de son père, Guillard de la Vacherie, préféra, au sens littéral du mot, céder du terrain.

Guillard se défendit un peu pour la forme, et protesta contre l'accusation, affirmant que le No 23 avait bien été payé ce qu'il valait. Ce nonobstant, "désirant éviter à la demoiselle Pérusse d'Escars l'action proposée, et donner des marques de désintéressement", il accepta un compromis. Les parties choisirent chacune un expert. L'un s'appelait Théophile Chauroy, de Coussay-les-Bois. L'autre portait un nom de consonance très révolutionnaire: Guillotin. Ils se mirent d'accord pour que les réclamants obtinssent un supplément de 300 livres en assignats sur le prix de vente de 1791. François Martin, qui n'avait pas oublié la promesse a lui faite par le marquis, trois ans auparavant, réussit enfin à avoir

87. Archives Nationales. W 406. 
les 20 boisselées de brandes devant lui permettre un accès facile à sa métairie. Elles furent prises sur le territoire du No 23. Le grignotage de l'œuvre d'Amirault était commencé.

Depuis ses mésaventures avec ses deux métairies, Guillard de la Vacherie avait fait, dans la connaissance de la mentalité paysanne et la pratique des marchandages, de sérieux progrès. Il y paraît à la lecture d'une clause introduite au sein de l'acte traité avec Martin et Charaudeau. Clause qu'un novice en affaires n'eât sûrement jamais trouvée.

Puisque l'on vient d'admettre la révision du premier article figurant à la vente de 1791 , c'est-à-dire du No 23 , on doit accepter, en vertu du même théorème, que le prix du deuxième article, c'est-àdire du No 19, soit également reconsidéré. La demoiselle Pérusse d'Escars, vendeur, est parfaitement fondée à se retourner, elle aussi, contre l'acheteur, Charaudeau, et à lui demander un supplément. Elle en a le droit. Néanmoins, elle n'entend pas en profiter. Ce droit, son fondé de pouvoirs tient moins à l'exercer qu'à le monnayer, de manière à compenser en partie la perte sèche des 300 livres versées tout à l'heure. Il va le vendre, comme on vendait alors le droit de reméré qui était aussi, à un autre titre, une menace suspendue sur la tête de l'acheteur. Donc, il "cède et transporte à Martin et Anne Hébert l'action en restitution, pour l'exercer à leur risque, péril et fortune, sur le prix de l'habitation ci-après quand ils aviseront contre François Charaudeau et sa femme, acquéreurs du No 19." Pour ce droit et les 20 boisselées de brandes, on convient d'un prix global de 800 livres $^{88}$.

Il est difficile d'éviter ici une question dont on sait d'avance que la réponse n'est pas dans les textes. Est-ce bien Guillard de la Vacherie qui avait eu l'idée astucieuse de transformer la réclamation de Martin en un boomerang qui se retournait maintenant contre son auteur? L'ex-officier du Normandie Infanterie a-t-il trouvé cela tout seul ? Ou quelqu'un, maniant en virtuose la science juridique l'a-t-il conseillé ? Cherchons à qui l'affaire profite, et nous serons peut-être sur la voie.

L'épine introduite malignement dans le No 19 pouvait devenir, les circonstances aidant, un danger sérieux. Le droit acheté par

88. Minutes Amirault. 
François Martin était une source de procès dont on ne pouvait prévoir le dénouement. Aussi bien, trois mois après, jour pour jour, c'est-à-dire le 24 thermidor an II, Charaudeau et Marie Hébert se débarrassaient-ils de leur fâcheux domaine. Ils n'eurent pas à chercher bien loin pour trouver un acheteur. Voici revenir, sur le devant de la scène, le syndic Texier-Latouche, qui va recueillir le fruit de ces opérations subtiles, alors que nous pensions, depuis un moment, l'avoir perdu de vue.

Il était, rappelons-le, locataire du No 19 pour 9 ans, depuis la Saint-Martin 1785, et c'est précisément cette année 1794 qu'expirait son bail. De plus, ce No 19 touchait aux No 17 et 18, provenant de Berbudeau. Le dessein de Texier, en l'achetant, va nous apparaître clairement.

Tout d'abord, le syndic arrache l'écharde qui faisait, du No 19 , un lot indésirable. Charaudeau et sa femme vendent leur domaine acadien en garantissant l'acquéreur "de toute action en restitution qui pourrait être intentée par un tiers pour cause de lésion, promettant, si pareille action avait lieu, d'en faire leur propre affaire ou s'en défendre ainsi qu'ils aviseront contre l'auteur de la dite action". La menace est levée, et l'on peut faire confiance au licencié ès lois pour qu'elle ne vienne jamais le troubler.

L'acheteur fait remarquer que les bâtiments, inhabités depuis longtemps, sont délabrés, que le puits est comblé, et qu'une dépense de 600 livres est à prévoir pour la remise en état. L'ensemble est vendu 2,130 livres dont 70 déjà versées et 910 payées comptant. Le reste acquittable en trois termes, sans intérêt ${ }^{89}$. A noter que le No 19 comprend, au moment de la vente, sur ses 176 boisselées réglementaires, 36 en jardin et en labour. C'est peu, à côté des 130 boisselées qu'avait défrichées Jean-Baptiste Hébert au No 23, mais c'est suffisant pour l'usage que Texier veut faire de sa nouvelle acquisition.

Il va purement et simplement la fusionner avec son No 17, faisant, des deux domaines, une seule et même exploitation. Nous n'avons pas la surface totale des labours, mais le cheptel nous prouve, sans équivoque possible, que Texier crée là une borderie à l'image du Charbon blanc ou de la Grève, les deux propriétés de son père,

89. Minutes Amirault. 
puisqu'elle est exploitée avec 2 bœufs, 1 charrette, 1 charrue, 1 herse; qu'il utilise ses vastes étendues de brandes à élever un troupeau de moutons atteignant le chiffre, considérable pour la région, de 243 unités et qu'il ne prévoit pas, pour l'instant, de nouveaux défrichements.

La borderie ainsi organisée est louée, le 15 messidor an VIII, à Louis Testard et Jeanne Auzillault, laboureurs de la région, pour sept ans, commencés le ler germinal précédent. Texier fait l'avance de 40 boisseaux de froment et 58 d'avoine. Le bail à colonnage, avec partage à mi-fruits, appartient au type classique. Il n'est plus question de progression dans le taux des fermages, comme en 1785. Le nouveau domaine est stabilisé ${ }^{90}$.

Dès lors, deviennent disponibles les brandes qui entourent la borderie en grande quantité. Texier se débarrasse de 104 boisselées 2 chaînées $2 / 3$ dont il n'a que faire, en les vendant, l'année suivante, à Cuisinier de l'Isle, propriétaire à Saint-Savin, qui les paye, les II nivôse an IX, 504 francs en numéraire métallique. Nous pouvons les retrouver, sur le plan de 1793, sans difficulté. Elles occupent deux parcelles marquées $17,18 \mathrm{C}$ et $19 \mathrm{~B}$. La confrontation des articles est tout à fait précise à cet égard.

Achat et vente. Ces deux activités de Texier-Latouche ne viennent pas seulement modifier la configuration des domaines. Il y a plus. La fusion de deux métairies, suivie d'un démembrement, remet en jeu le principe même de la colonie. En fait, c'est le mode d'occupation, et, par suite, d'exploitation du sol, qui est désormais envisagé sous un autre angle. Le remaniement des 176 boisselées de brandes, constituant l'unité de culture et considérées comme la propriété-type, est commencé. Nous le verrons gagner de proche en proche, par une véritable réaction en chaîne, et s'accélérer à une cadence accrue lorsque la Révolution agricole fera sentir ses premiers effets. Au terme de cette vaste transformation d'ordre spatial et d'ordre économique, nous trouverons l'openfield installé sur les carrelages d'Amirault, dont les lignes maîtresses apparaissent encore, aisément reconnaissables, sur la carte aérienne la plus récente.

90. Ibid. 
Ainsi, les projets de Pérusse d'Escars, et la répartition des terres qui s'en inspira, n'ont pu vivre longtemps. C'est en cela, aussi, que la colonie acadienne du Poitou se solde par un échec, mais un échec utile, celui-là, puisqu'il devait former un paysage rural a l'image des pays environnants et animé d'une vie semblable. L'ilôt construit de façon spéciale, au cœur des brandes, s'est bien vite laissé pénétrer par les contraintes collectives qui régissaient, depuis des siècles, le Châtelleraudais, et, là comme ailleurs, ont rapidement imposé leurs lois. Il était bon de préciser que c'est le syndic des Acadiens qui leur a, toute grande, ouvert la porte.

\section{LE PLAN D'AMIRAULT.}

LE DOMAINE DU SYNDIC DANS LA COLONIE ACADIENNE D'APRÈS

Les habitations No 17 et 18 appartenant à TexierLatouche se font vis-à-vis, de part et d'autre de la Ligne, immédiatement après le carrefour du Nord-Ouest. Le No 17 est au Sud de la Ligne, le No 18, au Nord.

La formation du domaine acadien du syndic a eu lieu en quatre étapes:

1o - en 1793. - Le bornoiement confère à TexierLatouche, comme à tout propriétaire de la colonie, une surface de 176 boisselées (17 hectares, 4064). Celle-ci est formée de 3 pièces.

La première, numérotée $17 \mathrm{~A}$ par l'arpenteur, mesure 142 boisselées 12 chaînées 3/4. Elle est située au Sud de la maison 17 . Sa forme est celle d'un rectangle allongé, limité au Nord-Ouest par le chemin de la Bironnerie.

La deuxième, de forme très irrégulière, est séparée par la Ligne de la précédente qu'elle prolonge au Nord, en englobant la maison 18. Elle mesure 174 boisselées 5 chaînées 1/4.

La troisième, enfin, numérotée $1718 \mathrm{C}$, est enclavée entre les terres $19 \mathrm{~B}, 39 \mathrm{~A}, 21 \mathrm{~B}, 24 \mathrm{~B}$. Nettement plus petite que les deux autres, elle mesure seulement 34 boisselées 12 chaînées.

20 - le 24 thermidor an II - Texier-Latouche achète les terres du No 19, dont la maison, au Sud de la Ligne, vient après le No 18. Le domaine est fait de deux tronçons, l'un numéroté $19 \mathrm{~A}$, de forme coudée, mesurant 106 boisselées 12 chaînées 1/2; l'autre numéroté $19 \mathrm{~B}$, mesurant 69 boisselées 12 chaînées 2/3. Il était locataire de ces terres, avoisinant les siennes, depuis la Saint-Martin 1785. 
Nous avons souligné les contours des domaines No 17 et 18, et hachuré le No 19.

3o - le 15 messidor an VIII - Il fusionne les parcelles 17 A et 19 A qui se touchent, pour faire une borderie, dont l'habitation sera la maison No 17.

40 - le II nivôse an IX - Il se débarrasse des deux tronçons $17,18 \mathrm{C}$ et $19 \mathrm{~B}$ demeurés en brandes, et qui, rangés côte à côte, enclavés au milieu d'autres terres, sont nettement isolés par rapport au noyau du domaine.

On remarquera: au Nord-Ouest des habitations du syndic, dans la patte d'oie, la terre des Vachons, de Chauvigny, sur laquelle on avait empiété, longeant la parcelle $17 \mathrm{~A}$ au Nord-Ouest, le chemin de la Bironnerie, dont la présence explique l'intérêt que Texier-Latouche prenait à cette métairie.

Nous avons conservé l'orientation du plan d'Amirault, qui accuse un certain décalage par rapport à l'orientation conventionnelle des cartes.

Rappelons que la boisselée, mesure de Châtellerault, vaut 9 ares, 89 , et la chaînée 65 mètres carrés, 934.

(à suivre)

Pierre Massé,
Paris, France

Histoire du Canada français, par le Chanoine Lionel Groulx (2e tome) Fin du régime français $(1713-1760)$. On est prié de prendre note que ce deuxième tome de l'Histoire du Canada français (304 pages) paraitra vers le 15 octobre. En vente à l'Action Nationale, 986 est, rue Rachel, Montréal, et dans toutes les bonnes librairies. Prix: $\$ 2.50$. Il reste encore quelques exemplaires du premier tome. 